\title{
Features of the microwave fields' influence on cereals
}

\author{
Dmitrii Poletaev ${ }^{1, *}$, Anatoliy Shadrin ${ }^{1}$, Alexander Nudga $^{1}$, Bohdan Sokolenko ${ }^{2}$, \\ Svetlana Chmeleva ${ }^{3}$, and Yulia Shabanova ${ }^{1}$ \\ ${ }^{1}$ V.I. Vernadsky Crimean federal university, department of radiophysics and electronics, 295007, 4 \\ Academician Vernadsky av., Simferopol, Russia \\ ${ }^{2}$ V.I. Vernadsky Crimean federal university, department of general physics, 295007, 4 Academician \\ Vernadsky av., Simferopol, Russia \\ ${ }^{3}$ V.I. Vernadsky Crimean federal university, department of botany and plant physiology and \\ biotechnology, 295007, 4 Academician Vernadsky av., Simferopol, Russia
}

\begin{abstract}
In this paper we describe a study of microwave fields' influence on cereals. A change in the energy of germination and germination was noted. Descriptions of the methods of the experiments are presented. Methods for determining the wavelength of microwave radiation for irradiating cereal crops are proposed. Also hypothesized about the causes of the change in energy of germination and germination of cereals under the influence of the microwave field.
\end{abstract}

\section{Introduction}

Cereals are important crops for agro-industrial complexes of modern countries. They are used in the manufacture of food and feed. Increasing the yield of cereal crops without the use of additional chemical fertilizers and maintaining the existing irrigation regime is an urgent task. However, direct methods for measuring yield require a very long preparation time (preparing images, sowing, ripening, harvesting). Much more expressive are experiments on measuring the germination and growth energy of cereals, directly related to their productivity.

In works $[1,2]$ the mechanisms of the action of microwave electromagnetic fields on biological objects are discussed. The mechanisms are as follows: thermal, resonant and field. The degree of action of the thermal mechanism [1] is determined mainly by the magnitude of the power of the electromagnetic wave. It consists in heating a biological object subjected to irradiation with electromagnetic waves. The resonance mechanism [1] is detected at certain wavelengths of the electromagnetic wave, regardless of its power. This allows to significantly save energy, minimizing exposure time and its intensity. In the field action mechanism, the leading role is played by the electric field strength modulus [1]. With increasing intensity of the electromagnetic wave, the dominance of the field mechanism increases.

*Corresponding author: poletaevda@cfuv.ru 
It is advisable to determine the wavelength and exposure time interval of microwave exposure which lead to higher yields.

The aim of the work is to propose a criterion for determining the optimal wavelength of microwave radiation affecting cereal crops, and the time interval of such exposure.

\section{Methodology of the experiment}

Investigated cereals was barley. For the experiment, the grains were prepared for sowing. This operation included sampling without visible damage and their disinfection with a solution of $1 \%$ potassium permanganate for 10 minutes. Experiments on the effects of microwave electromagnetic fields on cereal culture were carried out in two frequency ranges: $2.0 \mathrm{GHz}-4.0 \mathrm{GHz}$ and $37.5 \mathrm{GHz}-50 \mathrm{GHz}$. The setup diagrams are shown for both frequency ranges in fig. 1 and fig. 2 , respectively.



Fig. 1. Installation diagram for irradiating cereal crops with an electromagnetic wave in the frequency range $2.0 \mathrm{GHz}-4.0 \mathrm{GHz}$.



Fig. 2. Installation diagram for irradiating cereal crops with an electromagnetic wave in the frequency range $37.5 \mathrm{GHz}-50 \mathrm{GHz}$.

In the fig. 1 and fig. 2 are indicated: $\mathrm{K}-$ klystron microwave generator; $\mathrm{C}-$ section of radiotransparent in the range from 2 to $50 \mathrm{GHz}$ material with placed inside it cereals. The power of the klystron microwave generator in both cases did not exceed $10 \mathrm{~mW}$.

In the frequency range $2.0 \mathrm{GHz}-4.0 \mathrm{GHz}$, a portion of 100 prepared grains, in a random orientation, was placed in a section, uniformly filling the cross section of a rectangular waveguide with dimensions 72 by $34 \mathrm{~mm}$. Then, the frequency of the electromagnetic wave with a minimum VSWR was selected for a specific portion of the cereal culture [3]. This frequency corresponds to the largest absorption of electromagnetic energy in the irradiated object. Then, an exposure to an electromagnetic wave with this frequency on the grains was carried out, the duration of the exposure changed. 
In the frequency range $37.5 \mathrm{GHz}-50 \mathrm{GHz}$, a portion of 20 prepared grains was uniformly placed in the section located at the open end of a rectangular waveguide. In this case, the grains were oriented in such way that the conditional line drawn along the grain length appeared parallel to the smaller side of the waveguide. The placement of the specified cereal culture inside the waveguide in this frequency range is difficult, due to its compact size. Then, based on a comparison of the linear length of the grain embryo, the frequency of the electromagnetic wave was chosen. The linear wavelength was determined during microscopic studies of a specific cereal crop. For this, each grain of 10 prepared grains was cut and the length of the embryo and its orientation relative to the shell were measured under a microscope. Then, the data on the length of the embryo and its orientation were averaged. The length of the electromagnetic wave was chosen in such a way that its quarter was equal to the linear length of the grain germ, which corresponds to the optimal matching of the receiving antenna [4]. Then, an exposure to an electromagnetic wave with this frequency on the grains was carried out, the duration of exposure changed.

Measured parameters of cereal crops were: germination and germination energy. Germination energy was calculated as the percentage of germinated seeds on the 3rd day after sowing. Germination was calculated as the percentage of grains with a root's length which exceeds the length of the grain.

\section{Results and discussion}

The fig. 3 shows data on the germination energy and germination of barley upon irradiation at a frequency of $3.509 \mathrm{GHz}$, corresponding to the minimum VSWR, with different durations: $0 \mathrm{~s}, 60 \mathrm{~s}, 300 \mathrm{~s}$ and $900 \mathrm{~s}$. Most likely, the resonant mechanism of influence prevails in this range, since an increase in the germination and germination energy is observed at small values of the electromagnetic wave power. Apparently, local heating by an electromagnetic wave activates metabolic processes and acceleration of grain growth. A longer period of exposure to the microwave field leads to an increase in germination energy and germination. However, it is also necessary to analyze the growth rate of irradiated grains. Such experiments planned in the future.



Fig. 3. Data on the germination and germination energy when exposed to an electromagnetic wave with a frequency of $3.509 \mathrm{GHz}$.

The average value of the linear length of the grain embryo is $2 \mathrm{~mm}$, which corresponds to a frequency of $37.5 \mathrm{GHz}$. The fig. 4 shows data on the germination energy and germination of barley upon irradiation at a frequency of $37.5 \mathrm{GHz}$ with different durations: $0 \mathrm{~s}, 60 \mathrm{~s}$, $300 \mathrm{~s}$ and $900 \mathrm{~s}$. Most likely, in the frequency range $37.5 \mathrm{GHz}-50 \mathrm{GHz}$, the resonant mechanism of the influence of electromagnetic fields on biological objects prevails. Indeed, the correspondence of the linear dimensions of grain embryo of a quarter of the wavelength 
of incident radiation allows us to consider these objects as matched receiving antennas. Apparently, the local heating of the grain by an electromagnetic wave leads to the activation of metabolic processes and acceleration of growth rates. A longer period of time of exposure to the microwave field leads to an increase in the energy of germination and germination. However, it is also necessary to analyze the growth rate of irradiated grains. Such experiments planned in the future.

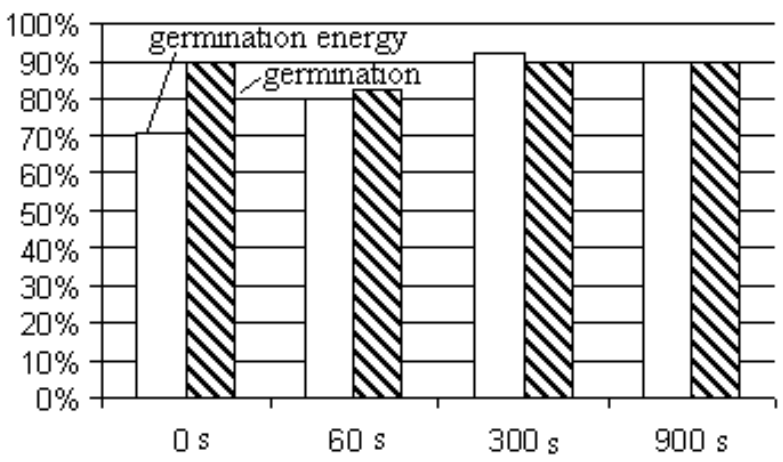

Fig. 4. Data on the germination and germination energy when exposed to an electromagnetic wave with a frequency of $37.5 \mathrm{GHz}$.

\section{Acknowledgments}

This work was supported by a scholarship of the Russian Federation's President to young scientists and graduate students SP-745.2019.4.

\section{References}

1. V. Starostenko, Radiofizika i elektronika. 7, 158 (2002)

2. N. Devyatkov, M. Golant, O. Betskiy, Millimeter waves (Radio i svyaz, Moscow, 1991)

3. C. Balanis, Antenna theory: analysis and design (Harper \& Row, New York, 1982)

4. S. Schelkunoff, H. Friis, Antennas: theory and practice (Wiley, New York, 1952) 